\title{
A Hamiltonian approach to fairly low and fairly long gravity waves
}

\author{
W.A. VAN DER VEEN ${ }^{1}$ and F.W. WUBS ${ }^{2}$ \\ ${ }^{1}$ Centre for Mathematics and Computer Science, P.O. Box 4079, 1009 AB Amsterdam, The Netherlands \\ ${ }^{2}$ Department of Mathematics, University of Groningen, P.O. Box 800, 9700 AV Groningen, The Netherlands (for \\ correspondence)
}

Received 10 November 1993; accepted in revised form 19 September 1994

\begin{abstract}
The propagation of nonlinear dispersive gravity waves in an inviscid irrotational fluid can be described by a Hamiltonian system. The canonical equations contain a boundary integral which is computationally expensive. However, for fairly low and fairly long waves an approximation can be made that gives rise to the solution of computationally more attractive Helmholtz-type equations. In an earlier attempt by Broer et al. $[4,6]$ canonical equations were derived that are stable for all wavenumbers. However, two Helmholtz-type equations need to be solved per right-hand side evaluation. In this paper, canonical equations are presented with the same qualities, but now only once per right-hand side evaluation a Helmholz-type equation needs to be solved, which is optimal.
\end{abstract}

\section{Introduction}

We consider irrotational nonlinear dispersive gravity waves in an inviscid incompressible fluid. The Boussinesq equations, we are aiming at, describe the propagation of fairly low and fairly long waves and take into account the effect of amplitude and frequency dispersion. Such waves occur in harbors and coastal regions. For long waves the frequency dispersion is small and such waves are usually modeled by the shallow-water equations. If the waves are low, the amplitude dispersion is small and they can be described by a linear set of equations. In order to indicate how low or how long a wave is, we introduce the parameters $\varepsilon$ and $\mu$, defined by $\varepsilon=a / h_{0}$ and $\mu=\left(h_{0} / L\right)^{2}$. Here, $a, h_{0}$ and $L$ are characteristic values of the wave amplitude, depth and wavelength, respectively.

In the process of deriving Boussinesq equations, the flow quantities are expanded in power series of $\varepsilon$ and $\mu$ and we seek a first-order (the error is $O\left(\varepsilon^{2}, \varepsilon \mu, \mu^{2}\right)$ ) approximation of the nonlinear wave equations. Hence, these parameters should be small in order to justify the neglect of the higher-order terms. These equations are not unique and we try to find a set with favourable stability properties.

Due to round-off errors, the numerical solution of a partial differential equation gives rise to short waves, which should not disturb the solution significantly or lead to an unstable computation. A necessity for the construction of a stable numerical model is a stable continuous model. Hence, also the continuous Boussinesq equations should be stable for short waves far away from the regime for which they have been derived.

Positive-definite Hamiltonians give canonical equations which are stable for all waves. For this reason we start from the general Hamiltonian formulation of water waves, which has this property (see Section 2). The Hamiltonian represents the total energy of the fluid and its canonical variables are $\zeta$ and $\phi(x, y, t)=\Phi(x, y, \zeta(x, y, t))$. Here $\zeta$ and $\Phi$ denote the waterelevation and the velocity potential, respectively. To obtain stable Boussinesq equations, the Hamiltonian is approximated by a simpler form, which is only valid for small $\mu$ and $\varepsilon$ but is still positive definite. This approach has already been used by Broer et al. $[4,6]$. 
Mooiman has derived and implemented a set of Boussinesq equations based on such a Hamiltonian [7, 8]. The solution of the Helmholtz-type equations dominates the run time of the model. Van der Ploeg [9] compared a variety of numerical solution techniques for these equations, which resulted in a speed-up of an order of magnitude. However, this part is still dominating the run time. In fact, in Mooiman's model in each right-hand side evaluation two times a Helmholtz-type equation has to be solved in which the operator is non-symmetric. In Section 3, we derive an alternative positive-definite first-order approximation to the Hamiltonian. The corresponding canonical equations are presented in Section 4. In the implementation of this alternative only one Helmholtz-type equation needs to be solved per right-hand side evaluation and the corresponding matrix is symmetric. The latter is an additional advantage, because it simplifies the solution process. These favourable properties are due to a better choice of the approximate Hamiltonian.

\section{The Hamiltonian for nonlinear gravity waves}

In this section, we will present a short derivation of the equations and show that they have a Hamiltonian structure.

\subsection{THE EQUATIONS}

We consider nonlinear dispersive gravity waves in an incompressible and inviscid fluid. The propagation of these waves is described by the continuity equation

$$
u_{x}+v_{y}+w_{z}=0
$$

and the Euler equations

$$
\begin{gathered}
u_{t}+u u_{x}+v u_{y}+w u_{z}+\frac{1}{\rho} p_{x}=0 \\
v_{t}+u v_{x}+v v_{y}+w v_{z}+\frac{1}{\rho} p_{y}=0 \\
w_{t}+u w_{x}+v w_{y}+w w_{z}+\frac{1}{\rho} p_{z}=-g
\end{gathered}
$$

In these equations, $u$ and $v$ are the horizontal velocities in $x$ - and $y$-direction, respectively, $w$ is the vertical velocity (in $z$-direction), $p$ is the pressure, $\rho$ is the density of the homogeneous incompressible fluid, and $g$ is the gravitational acceleration.

We assume that the pressure outside the fluid is constant and equals $p_{0}$. In the Euler equations only the pressure gradient is involved. Hence, we can take $p=p_{0}=0$ at the free surface which is the so-called dynamic boundary condition. Furthermore, it is assumed that a particle on the free surface remains there. This yields the kinematic boundary condition at the free surface

$$
\zeta_{t}+u \zeta_{x}+v \zeta_{y}-w=0, z=\zeta(x, y, t)
$$

Finally the bottom is impermeable. Hence, we must require that the normal velocity at the bottom vanishes

$$
u h_{x}+v h_{y}+w=0, z=-h(x, y)
$$


In these equations, $z=0, z=-h(x, y)$ and $z=\zeta(x, y, t)$ give the position of the still water-level, the bottom and the free surface, respectively. We suppose that $u^{2}+v^{2}+w^{2} \rightarrow 0$ as $x^{2}+y^{2} \rightarrow \infty$. Hence, there is no main flow.

Furthermore we assume that the flow is irrotational. In this case the velocity can be written as the gradient of a scalar function. This function is called the velocity potential and is denoted by $\Phi$. Hence, we have

$$
u=\Phi_{x}, v=\Phi_{y}, w=\Phi_{z} .
$$

The boundary-value problem posed above can be reformulated in terms of $\Phi$ as follows

$$
\begin{aligned}
\Delta \Phi+\Phi_{z z} & =0,-h(x, y)<z<\zeta(x, y, t) \\
\Phi_{t}+\frac{1}{2}\left(|\nabla \Phi|^{2}+\Phi_{z}^{2}\right)+g \zeta & =0, z=\zeta(x, y, t) \\
\zeta_{t}+\nabla \Phi \cdot \nabla \zeta-\Phi_{z} & =0, z=\zeta(x, y, t) \\
\nabla \Phi \cdot \nabla h+\Phi_{z} & =0, z=-h(x, y)
\end{aligned}
$$

where $\nabla$ and $\Delta$ denote the 2-dimensional gradient and Laplace operator, respectively. This notation will be used throughout the paper. Because there is now main flow, we require that $\nabla \Phi$ vanishes as $x^{2}+y^{2} \rightarrow \infty$. Only gradients of $\Phi$ are involved in the equations so, in order to make it unique, we also require that $\Phi$ vanishes as $x^{2}+y^{2} \rightarrow \infty$. For future use, we also give here the linear dispersion relation, which is obtained from a Fourier transform of the linearized equations with horizontal bottom (see also [10]). It is given by

$$
c^{2}=\frac{g}{k} \tanh \left(h_{0} k\right)
$$

Equation (5) shows how the phase velocity $c$ depends on the depth $h_{0}$ and the wave number $k$ of a plane wave with velocity potential

$$
\cosh \left[k\left(z+h_{0}\right)\right] \exp \left[i\left(k_{1} x+k_{2} y-k c t\right)\right]
$$

where $k=\sqrt{k_{1}^{2}+k_{2}^{2}}$.

\subsection{HAMILTONIANS, CANONICAL VARIABLES AND EQUATIONS}

Many of the systems of continuum mechanics can be formulated (see [1]) in a special form

$$
\begin{aligned}
& \frac{\partial p_{i}}{\partial t}=-H_{q_{i}}(P, Q, t) \\
& \frac{\partial q_{i}}{\partial t}=H_{p_{i}}(P, Q, t)
\end{aligned}
$$

where $H$ is the so-called Hamiltonian density. These equations can be deduced from the variational equation

$$
\delta \iint_{\Omega}\left(P^{T} \frac{\mathrm{d} Q}{\mathrm{~d} t}-H\right) \mathrm{d} t \mathrm{~d} \Omega=0
$$

where $\Omega$ is the spatial domain on which the equations are defined. Here $P=\left(p_{1}, . ., p_{n}\right)$ and $Q=\left(q_{1}, . ., q_{n}\right)$ are scalar fields and fully determine the state of the system. It is customary 
to call $P$ and $Q$ the canonical variables. Integrating $H$ over $\Omega$ yields the Hamiltonian $\mathcal{H}$. In general for conservative systems the Hamiltonian $\mathcal{H}$ is equal to the total energy of the system. The equations (6) and (7) are the so-called canonical equations corresponding to the Hamiltonian $\mathcal{H}$.

The canonical equations corresponding to a time-independent and positive-definite Hamiltonian are such that the zero state $(P \equiv Q \equiv 0)$ is stable in the sense of Lyapunov [6]. We will briefly explain what this means. Suppose there exist positive constants $c_{1}$ and $c_{2}$ such that for all perturbations $\tilde{P}$ and $\tilde{Q}$ holds

$$
c_{1}\left(d_{1}(\tilde{P})+d_{2}(\tilde{Q})\right) \leq \mathcal{H}(\tilde{P}, \tilde{Q}) \leq c_{2}\left(d_{1}(\tilde{P})+d_{2}(\tilde{Q})\right)
$$

where $d_{i}$ is some norm, then we call the Hamiltonian positive definite (if $c_{1}=0$, then we call it positive). Due to the fact that $\mathcal{H}$ is independent of time we have for any $t_{1}>t_{0}$ that

$$
c_{1}\left(d_{1}(\tilde{P})+d_{2}(\tilde{Q})\right)_{t=t_{1}} \leq \mathcal{H}(\tilde{P}, \tilde{Q})_{t=t_{1}}=\mathcal{H}(\tilde{P}, \tilde{Q})_{t=t_{0}} \leq c_{2}\left(d_{1}(\tilde{P})+d_{2}(\tilde{Q})\right)_{t=t_{0}}
$$

Hence, the growth of the perturbations is bounded and thereby the zero state is stable.

\subsection{FORMULATING THE HAMILTONIAN SYSTEM}

The functions $\phi(x, y, t)=\Phi(x, y, \zeta(x, y, t))$ and $\zeta(x, y, t)$ fully determine the motion. Given $\zeta$ and $\phi$, then $\Phi$ can be computed by solving for fixed time the boundary value problem given by Equation (1) with boundary conditions (4) and $\Phi(x, y, \zeta)=\phi(x, y)$. Once $\Phi$ is known, the time evolution of $\phi$ and $\zeta$ is found from (2) and (3). In fact the equations (1)-(4) can be written explicitly in a boundary integral formulation in terms of $\phi$ and $\zeta$, but this leads to a computationally expensive numerical model. In the following we will show that the boundary-value problem (1)-(4) can be reformulated as a Hamiltonian system with a Hamiltonian $\mathcal{H}$ being the total energy $E_{k}+E_{p}$ and canonical variables $\phi$ and $\zeta$. For more theoretical background see $[2,3]$. The kinetic and potential energy are given by

$$
E_{k}=\frac{1}{2} \rho \int_{-\infty}^{\infty} \int_{-\infty}^{\infty} \int_{-h}^{\zeta}\left(|\nabla \Phi|^{2}+\Phi_{z}^{2}\right) \mathrm{d} z \mathrm{~d} y \mathrm{~d} x
$$

and

$$
E_{p}=\frac{1}{2} \rho \int_{-\infty}^{\infty} \int_{-\infty}^{\infty} g \zeta^{2} \mathrm{~d} y \mathrm{~d} x
$$

respectively. In view of our previous observation $\mathcal{H}$ is a function of $\phi$ and $\zeta$. Hence, we have to show that the boundary value problem is equivalent to the Hamiltonian system

$$
\begin{aligned}
& \rho \frac{\partial \phi}{\partial t}=-H_{\zeta} \\
& \rho \frac{\partial \zeta}{\partial t}=H_{\phi}
\end{aligned}
$$

Here $H_{\zeta}$ and $H_{\phi}$ are implicitly defined in terms of Gateaux derivatives by

$$
\begin{aligned}
\left(H_{\phi}, v\right) & =\delta \mathcal{H}((\phi, \zeta) ;(v, 0)) \\
\left(H_{\zeta}, v\right) & =\delta \mathcal{H}((\phi, \zeta) ;(0, v))
\end{aligned}
$$


where $v$ is an arbitrary function and the inner product is given by

$$
(v, w)=\int_{-\infty}^{\infty} \int_{-\infty}^{\infty} v w \mathrm{~d} x \mathrm{~d} y
$$

Now

$$
\delta \mathcal{H}((\phi, \zeta) ;(v, 0)) \equiv \lim _{\epsilon \rightarrow 0} \frac{\mathcal{H}(\phi+\epsilon v, \zeta)-\mathcal{H}(\phi, \zeta)}{\epsilon}
$$

Hence

$$
\begin{aligned}
\left(H_{\phi}, v\right) & =\lim _{\epsilon \rightarrow 0} \frac{1}{2} \rho \int_{-\infty}^{\infty} \int_{-\infty}^{\infty} \frac{1}{\epsilon}\left[\int_{-h}^{\zeta}|\nabla(\Phi+\epsilon V)|^{2}\right. \\
& \left.+(\Phi+\epsilon V)_{z}^{2}-|\nabla \Phi|^{2}-\Phi_{z}^{2} \mathrm{~d} z\right] \mathrm{d} x \mathrm{~d} y
\end{aligned}
$$

where $\Phi$ and $\Phi+\epsilon V$ are solutions of (1) with boundary condition (4) at the bottom and at the free surface $\Phi=\phi$ and $\Phi+\epsilon V=\phi+\epsilon v$, respectively. So we get

$$
\left(H_{\phi}, v\right)=\rho \int_{-\infty}^{\infty} \int_{-\infty}^{\infty} \int_{-h}^{\zeta}\left(\nabla \Phi \cdot \nabla V+\Phi_{z} V_{z}\right) \mathrm{d} z \mathrm{~d} x \mathrm{~d} y
$$

After applying Green's theorem and (1), it follows that

$$
\left(H_{\phi}, v\right)=\rho \int_{-\infty}^{\infty} \int_{-\infty}^{\infty}\left[\Phi_{n} V\right]_{z=-h}^{z=\zeta} \mathrm{d} S
$$

where $\mathrm{d} S$ is a surface element. Using the impermeability of the bottom (4), we obtain

$$
\begin{aligned}
\left(H_{\phi}, v\right) & =\rho \int_{-\infty}^{\infty} \int_{-\infty}^{\infty}\left[\Phi_{n} V\right]_{z=\zeta} \mathrm{d} S \\
& =\rho \int_{-\infty}^{\infty} \int_{-\infty}^{\infty} v\left[\Phi_{n}\right]_{z=\zeta} \sqrt{\left(1+\zeta_{x}^{2}+\zeta_{y}^{2}\right)} \mathrm{d} x \mathrm{~d} y
\end{aligned}
$$

This gives

$$
H_{\phi}=\rho\left[\Phi_{n}\right]_{z=\zeta} \sqrt{\left(1+\zeta_{x}^{2}+\zeta_{y}^{2}\right)}
$$

The outward normal $n$ on the surface is

$$
n=\left(-\zeta_{x},-\zeta_{y}, 1\right)^{T} / \sqrt{\left(1+\zeta_{x}^{2}+\zeta_{y}^{2}\right)}
$$

and this finally yields

$$
H_{\phi}=-\rho\left(\nabla \Phi \cdot \nabla \zeta-\Phi_{z}\right)_{z=\zeta}
$$

Indeed we see that the canonical equation (10), with right-hand side (12), is equivalent to Equation (3).

Now, we will vary $\zeta$ keeping $\phi$ fixed. From $\phi(x, y, t)=\Phi(x, y, \zeta(x, y, t), t)$ it follows that a variation $\epsilon v$ in $\zeta$ induces a variation $-\epsilon \Phi_{z} v$ in $\Phi$ at the surface. Hence,

$$
\begin{aligned}
\delta \mathcal{H}((\phi, \zeta) ;(0, v)) & \equiv \lim _{\epsilon \rightarrow 0} \frac{\mathcal{H}(\phi, \zeta+\epsilon v)-\mathcal{H}(\phi, \zeta)}{\epsilon} \\
& =\lim _{\epsilon \rightarrow 0} \frac{1}{2} \rho \int_{-\infty}^{\infty} \int_{-\infty}^{\infty} \frac{1}{\epsilon}\left[\int_{-h}^{\zeta+\epsilon v}|\nabla(\Phi+\epsilon V)|^{2}\right. \\
& \left.+(\Phi+\epsilon V)_{z}^{2}-|\nabla \Phi|^{2}-\Phi_{z}^{2} \mathrm{~d} z+g\left(2 \zeta \epsilon v+(\epsilon v)^{2}\right)\right] \mathrm{d} x \mathrm{~d} y
\end{aligned}
$$


where here $\epsilon V$ is due to the variation $-\epsilon \Phi_{z} v$ at the surface. This equation is equal to

$$
\begin{aligned}
\left(H_{\zeta}, v\right)= & \lim _{\epsilon \rightarrow 0} \frac{1}{2} \rho \int_{-\infty}^{\infty} \int_{-\infty}^{\infty} \frac{1}{\epsilon}\left[\int_{\zeta}^{\zeta+\epsilon v}\left(|\nabla \Phi|^{2}+\Phi_{z}^{2}\right) \mathrm{d} z\right. \\
& \left.+g\left(2 \zeta \epsilon v+(\epsilon v)^{2}\right)\right] \mathrm{d} x \mathrm{~d} y \\
& +\frac{1}{2} \rho \int_{-\infty}^{\infty} \int_{-\infty}^{\infty} \frac{1}{\epsilon}\left[\int_{-h}^{\zeta}|\nabla(\Phi+\epsilon V)|^{2}\right. \\
& \left.+(\Phi+\epsilon V)_{z}^{2}-|\nabla \Phi|^{2}-\Phi_{z}^{2} \mathrm{~d} z\right] \mathrm{d} x \mathrm{~d} y
\end{aligned}
$$

The first part is simple and the second part can be treated as before with Green's theorem. This yields

$$
\begin{aligned}
\left(H_{\zeta}, v\right)= & \frac{1}{2} \rho \int_{-\infty}^{\infty} \int_{-\infty}^{\infty}\left[\left(|\nabla \Phi|^{2}+\Phi_{z}^{2}\right)_{z=\zeta}+2 g \zeta\right] v \mathrm{~d} x \mathrm{~d} y \\
& +\rho \int_{-\infty}^{\infty} \int_{-\infty}^{\infty}\left[\Phi_{n} V\right]_{z=-h}^{z=\zeta} \mathrm{d} S
\end{aligned}
$$

and therefore

$$
H_{\zeta}=\rho\left[\frac{1}{2}\left(|\nabla \Phi|^{2}+\Phi_{z}^{2}\right)_{z=\zeta}+g \zeta-\left(\Phi_{z} \Phi_{n}\right)_{z=\zeta} \sqrt{\left(1+\zeta_{x}^{2}+\zeta_{y}^{2}\right)}\right]
$$

The last part is precisely the difference between $\phi_{t}$ and $\Phi_{t}$, i.e.

$$
\phi_{t}=\left(\Phi_{t}+\Phi_{z} \zeta_{t}\right)_{z=\zeta}=\left[\Phi_{t}+\left(\Phi_{z} \Phi_{n}\right) \sqrt{\left(1+\zeta_{x}^{2}+\zeta_{y}^{2}\right)}\right]_{z=\zeta}
$$

Hence, the canonical equation (9) with $H_{\zeta}$ given by (13) is equivalent to Equation (2).

\section{Approximating the Hamiltonian}

In order to be able to evaluate the quality of an approximation of the Hamiltonian, we write the problem in dimensionless form. We assume that all quantities can be written as power series expansions in $\varepsilon$ and $\mu$, where $\varepsilon$ and $\mu$ are defined by $\varepsilon=a / h_{0}$ and $\mu=\left(h_{0} / L\right)^{2}$. Here $h_{0}$, $a$ and $L$ are characteristic values of the depth, wave amplitude and wavelength, respectively. The parameters $\varepsilon$ and $\mu$ measure how low and how long a wave is. For fairly low and fairly long waves $\varepsilon$ and $\mu$ are small with respect to one. We will derive a Hamiltonian which is equal to the exact Hamiltonian up to $O\left(\varepsilon^{2}, \varepsilon \mu, \mu^{2}\right)$. Thus, we will obtain a first-order approximation.

\subsection{THE DIMENSIONLESS PROBLEM}

First, we will introduce the characteristic values of the involved variables. Thereafter, the equations in the new variables will be presented. Horizontal distances are compared to the characteristic wavelength $L$. Hence, the characteristic wave number $k_{0}$ can be chosen as $1 / L$. As the characteristic wave speed for these shallow-water waves is $c_{0}=\sqrt{g h_{0}}$, we find a characteristic time scale $T=L / c_{0}$. Moreover, the characteristic depth and wave amplitude are $h_{0}$ and $\varepsilon h_{0}$, respectively. Herewith, we introduced the following dimensionless quantities:

$$
\begin{aligned}
& \bar{x}=x / L, \quad \bar{y}=y / L, \quad \bar{t}=\left(L / c_{0}\right) t, \\
& \bar{k}=L k, \quad \bar{h}=h / h_{0}, \quad \bar{\zeta}=\zeta / \varepsilon h_{0}
\end{aligned}
$$


The characteristic value of $\Phi$ follows from the linearized version of (2) and is given by $\varepsilon c_{0} L$. Now, from the linearized version of (3), it follows that the characteristic value of $z$ is $L^{2} / h_{0}$. Hence, we have found the important dimensionless quantities

$$
\bar{z}=\frac{h_{0}}{L^{2}} z, \quad \bar{\Phi}=\frac{\Phi}{\varepsilon c_{0} L}
$$

With these values all the remaining quantities can be put in dimensionless form

$$
\bar{\Phi}_{\bar{x}}=\frac{1}{\varepsilon c_{0}} \Phi_{x}, \bar{\Phi}_{\bar{z}}=\frac{1}{\varepsilon c_{0} \sqrt{\mu}} \Phi_{z}, \overline{\mathcal{H}}=\frac{\mathcal{H}}{\rho \varepsilon^{2} g h_{0}^{2} L^{2}}
$$

Omitting all bars, the boundary-value problem (1-4) can be rewritten in dimensionless variables as follows:

$$
\begin{aligned}
\Delta \Phi+\mu \Phi_{z z} & =0,-\mu h(x, y)<z<\varepsilon \mu \zeta(x, y, t) \\
\Phi_{t}+\frac{1}{2} \varepsilon\left(|\nabla \Phi|^{2}+\mu \Phi_{z}^{2}\right)+\zeta & =0, z=\varepsilon \mu \zeta(x, y, t) \\
\zeta_{t}+\varepsilon \nabla \Phi \cdot \nabla \zeta-\Phi_{z} & =0, z=\varepsilon \mu \zeta(x, y, t) \\
\nabla \Phi \cdot \nabla h+\Phi_{z} & =0, z=-\mu h(x, y)
\end{aligned}
$$

The Hamiltonian written in these dimensionless variables is

$$
\mathcal{H}=\frac{1}{2} \int_{-\infty}^{\infty} \int_{-\infty}^{\infty}\left(\frac{1}{\mu} \int_{-\mu h}^{\varepsilon \mu \zeta}|\nabla \Phi|^{2}+\mu \Phi_{z}^{2} \mathrm{~d} z\right)+\zeta^{2} \mathrm{~d} x \mathrm{~d} y
$$

The dimensionless form of the dispersion relation (5) reads

$$
c^{2}=\frac{\tanh (\sqrt{\mu} k)}{\sqrt{\mu} k}
$$

This equation demonstrates for instance that for long waves on a shallow sea, hence for small $\sqrt{\mu}$, we have the typical shallow-water phase velocity $c=1$, or $\sqrt{g h_{0}}$ in dimensional form.

\subsection{REQUIREMENTS IMPOSED ON THE APPROXIMATION}

The approximation $\mathcal{H}_{a p p}$ to the Hamiltonian should describe the propagation of fairly low and fairly long waves well enough. To this end we require that $\mathcal{H}_{a p p}$ is a first-order approximation to the Hamiltonian $\mathcal{H}$ given by (16). Hence we demand that the difference $\mathcal{H}_{\text {app }}-\mathcal{H}$ consists of higher-order terms in $\varepsilon$ and $\mu$. To get useful results we assume that $\varepsilon$ and $\mu$ have the same order of magnitude. Hence, $\mathcal{H}_{\text {app }}$ should satisfy

$$
\mathcal{H}_{\text {app }}-\mathcal{H}=O\left(\varepsilon^{2}, \varepsilon \mu, \mu^{2}\right)
$$

In the process of numerically solving the canonical equations corresponding to $\mathcal{H}_{\text {app }}$, very short waves are created, due to truncation errors. These very short waves lie outside the Boussinesq regime and may not be correctly modeled by $\mathcal{H}_{\text {app }}$ which can lead to instabilities. In order to evade this problem, we require that $\mathcal{H}_{a p p}$ gives a stable canonical system. Hence, we require, similar to the discussion in Section 2.2 , that $\mathcal{H}_{a p p}$ is a positive definite function of $\nabla \phi$ and $\zeta$. Note that the value of $\phi$ itself is not important in the canonical equations. We summarize the requirements imposed on $\mathcal{H}_{a p p}$ :

1. $\mathcal{H}_{a p p}$ is a positive-definite function of $\nabla \phi$ and $\zeta$.

2. $\mathcal{H}_{\text {app }}-\mathcal{H}=O\left(\varepsilon^{2}, \varepsilon \mu, \mu^{2}\right)$ 


\subsection{THE APPROXIMATION}

Now we construct an approximation $\mathcal{H}_{a p p}$ to $\mathcal{H}=E_{k}+E_{p}$ which satisfies the requirements presented in the previous section. The potential energy $E_{p}$ is already in a suitable form. Hence, only $E_{k}$ remains to be approximated. To facilitate the approximation process, the kinetic energy $E_{k}$ is split in two parts

$$
E_{k}=E_{k, 0}+E_{k, s}
$$

where

$$
\begin{aligned}
& E_{k, 0}=\frac{1}{2 \mu} \int_{-\infty}^{\infty} \int_{-\infty}^{\infty} \int_{-\mu h}^{0}\left(|\nabla \Phi|^{2}+\mu \Phi_{z}^{2}\right) \mathrm{d} z \mathrm{~d} x \mathrm{~d} y \\
& E_{k, s}=\frac{1}{2 \mu} \int_{-\infty}^{\infty} \int_{-\infty}^{\infty} \int_{0}^{\varepsilon \mu \zeta}\left(|\nabla \Phi|^{2}+\mu \Phi_{z}^{2}\right) \mathrm{d} z \mathrm{~d} x \mathrm{~d} y
\end{aligned}
$$

In fact these are the kinetic energy below and above the still-water level, respectively. We will show that an $O\left(\varepsilon \mu, \mu^{2}\right)$ approximation of the exact Hamiltonian (16) in dimensionless form is given by

$$
\mathcal{H}_{\text {app }}=\frac{1}{2} \rho \int_{-\infty}^{\infty} \int_{-\infty}^{\infty}\left(\nabla \phi R \nabla \phi+\zeta^{2}\right) \mathrm{d} x \mathrm{~d} y
$$

where

$$
\begin{aligned}
R & =\frac{1}{\beta}\left((h+\varepsilon \zeta)^{-1}+\beta \mu A\right)^{-1}+\frac{\alpha}{\beta}(h+\varepsilon \zeta) \\
\beta & =\alpha+1
\end{aligned}
$$

in which the self-adjoint operator $A$ is defined by

$$
A f=-\frac{1}{6}(h \Delta f+\Delta(h f))+\frac{1}{3} h^{-1}|\nabla h|^{2} f
$$

with $f$ a sufficient smooth function on which $A$ operates. Recall that an operator is self-adjoint if $(u, A v)=(A u, v)$, where $(\cdot, \cdot)$ signifies the inner product defined in Equation (11). The value of the coefficient $\alpha$ should be nonnegative. A special choice of this coefficient will be treated at the end of this section.

As a first step, we will show that the intermediate approximation of the Hamiltonian

$$
\mathcal{H}_{0}=\frac{1}{2} \int_{-\infty}^{\infty} \int_{-\infty}^{\infty}\left(\nabla \phi R_{0} \nabla \phi+\varepsilon \zeta|\nabla \phi|^{2}+\zeta^{2}\right) \mathrm{d} x \mathrm{~d} y
$$

with

$$
R_{0}=\left(1-\frac{1}{2} \mu h^{2} \Delta\right)^{-1} h\left(1-\frac{1}{6} \mu h^{2} \Delta\right)
$$

is an $O\left(\varepsilon \mu, \mu^{2}\right)$ approximation of the exact Hamiltonian. This is proved in the following lemma in which the first part corresponds to the second term in (23); the second and third part to the first term. 
LEMMA 1. The following approximations hold

1. $E_{k, s}-\frac{1}{2} \int_{-\infty}^{\infty} \int_{-\infty}^{\infty} \varepsilon \zeta|\nabla \phi|^{2} \mathrm{~d} x \mathrm{~d} y=O(\varepsilon \mu)$

2. $E_{k, 0}-\frac{1}{2} \int_{-\infty}^{\infty} \int_{-\infty}^{\infty}\left(\nabla \Phi R_{0} \nabla \Phi\right)_{z=0} \mathrm{~d} x \mathrm{~d} y=O\left(\mu^{2}\right)$

3. $\frac{1}{2} \int_{-\infty}^{\infty} \int_{-\infty}^{\infty} \nabla \phi R_{0} \nabla \phi \mathrm{d} x \mathrm{~d} y-\frac{1}{2} \int_{-\infty}^{\infty} \int_{-\infty}^{\infty}\left(\nabla \Phi R_{0} \nabla \Phi\right)_{z=0} \mathrm{~d} x \mathrm{~d} y=O(\varepsilon \mu)$

Proof:

1. We will express both terms in the left-hand side in $\Phi$ at the surface. Subtraction leads immediately to the desired result.

On the interval $[0, \varepsilon \mu \zeta]$, we have for $|\nabla \Phi|^{2}+\mu \Phi_{z}^{2}$ the Taylor series expansion

$$
|\nabla \Phi|^{2}+\mu \Phi_{z}^{2}=\left(|\nabla \Phi|^{2}+\mu \Phi_{z}^{2}\right)_{z=\varepsilon \mu \zeta}+O(\varepsilon \mu)
$$

Herewith, $E_{k, s}$ can be written as

$$
E_{k, s}=\frac{1}{2} \int_{-\infty}^{\infty} \int_{-\infty}^{\infty} \varepsilon \zeta\left(|\nabla \Phi|^{2}+\mu \Phi_{z}^{2}\right)_{z=\varepsilon \mu \zeta} \mathrm{d} x \mathrm{~d} y+O\left(\varepsilon^{2} \mu\right)
$$

Because $\phi(x, y, t)=\Phi(x, y, \varepsilon \mu \zeta(x, y, t))$ we see that

$$
\nabla \phi=\left(\nabla \Phi+\varepsilon \mu \nabla \zeta \Phi_{z}\right)_{z=\varepsilon \mu \zeta}
$$

Hence,

$$
\frac{1}{2} \int_{-\infty}^{\infty} \int_{-\infty}^{\infty} \varepsilon \zeta|\nabla \phi|^{2} \mathrm{~d} x \mathrm{~d} y=\frac{1}{2} \int_{-\infty}^{\infty} \int_{-\infty}^{\infty} \varepsilon \zeta|\nabla \Phi|_{z=\varepsilon \mu \zeta}^{2} \mathrm{~d} x \mathrm{~d} y+O\left(\varepsilon^{2} \mu\right)
$$

Subtracting this expression from (24) yields

$$
E_{k, s}-\frac{1}{2} \int_{-\infty}^{\infty} \int_{-\infty}^{\infty} \varepsilon \zeta|\nabla \phi|^{2} \mathrm{~d} x \mathrm{~d} y=\frac{1}{2} \varepsilon \mu \int_{-\infty}^{\infty} \int_{-\infty}^{\infty} \zeta\left(\Phi_{z}^{2}\right)_{z=\varepsilon \mu \zeta} \mathrm{d} x \mathrm{~d} y+O\left(\varepsilon^{2} \mu\right)
$$

which proves Assertion 1.

2. By Green's theorem, we will transform the volume integral $E_{k, 0}$ given by (18) into a boundary integral. In the result, we need an approximation of $\Phi_{z}$ at the still-water level. This will be found using the impermeability condition at the bottom. In the truncation of the Taylor series used here, an approximation of $\Phi_{z}$ follows in terms of tangential differential and integral operators acting on $\Phi$ at the still-water level. After a simplifying step, the result follows upon substitution in the boundary integral.

Hence, we can write $E_{k, 0}$ by using Green's theorem as follows

$$
E_{k, 0}=\frac{1}{2} \rho \int_{-\infty}^{\infty} \int_{-\infty}^{\infty}\left[\Phi \Phi_{z}\right]_{z=0}+\left[\Phi \Phi_{n}\right]_{z=-\mu h} \mathrm{~d} x \mathrm{~d} y
$$

From $\Phi_{n}=0$ at $z=-\mu h(x, y)$ it follows that

$$
E_{k, 0}=\frac{1}{2} \rho \int_{-\infty}^{\infty} \int_{-\infty}^{\infty}\left(\Phi \Phi_{z}\right)_{z=0} \mathrm{~d} x \mathrm{~d} y
$$

Now, we have to remove the $z$-derivative of $\Phi$ in this expression. This can be accomplished by using the impermeability condition at the bottom (15). As a first step, we express the required 
quantities $\nabla \Phi$ and $\Phi_{z}$ at the bottom in terms of derivatives of $\Phi$ along the still-water level and in $\Phi_{z}(x, y, 0)$. Therefore, we form Taylor expansions and make use of the dimensionless Laplace equation (14) to get rid of as many $z$-derivatives as possible. In formulas this procedure reads

$$
\begin{aligned}
\nabla \Phi(x, y,-\mu h) & \approx \nabla \Phi(x, y, 0)-\mu h(\nabla \Phi)_{z}(x, y, 0)+\frac{1}{2}(\mu h)^{2}(\nabla \Phi)_{z z}(x, y, 0) \\
& =\left(1-\frac{1}{2}(\mu h)^{2} \Delta\right) \nabla \Phi(x, y, 0)-\mu h \nabla \Phi_{z}(x, y, 0)
\end{aligned}
$$

and

$$
\begin{aligned}
\Phi_{z}(x, y,-\mu h) \approx & \Phi_{z}(x, y, 0)-\mu h \Phi_{z z}(x, y, 0) \\
& +\frac{1}{2}(\mu h)^{2} \Phi_{z z z}(x, y, 0)-\frac{1}{6}(\mu h)^{3} \Phi_{z z z z}(x, y, 0) \\
= & \left(1-\frac{1}{2} \mu h^{2} \Delta\right) \Phi_{z}+h\left(1-\frac{1}{6} \mu h^{2} \Delta\right) \Delta \Phi(x, y, 0)
\end{aligned}
$$

In Appendix A, we consider an infinite expansion which may be of interest for higher-order approximations. As a second step, equations (27) and (28) are substituted in (15), which yields after some algebraic manipulations (see also Appendix A)

$$
\left(1-\frac{1}{2} \operatorname{div}\left(\mu h^{2} \nabla\right)\right) \Phi_{z}(x, y, 0)=-\operatorname{div}\left(h \nabla \Phi-\frac{1}{6} \mu h^{3} \nabla(\Delta \Phi)\right)_{z=0}+O\left(\mu^{2}\right)
$$

We define an approximation $C$ of $\Phi_{z}$ by

$$
\left(1-\frac{1}{2} \operatorname{div}\left(\mu h^{2} \nabla\right)\right) C=-\operatorname{div}\left(h \nabla \Phi-\frac{1}{6} \mu h^{3} \nabla(\Delta \Phi)\right)_{z=0}
$$

Hence,

$$
C-\Phi_{z}(x, y, 0)=O\left(\mu^{2}\right)
$$

and an $O\left(\mu^{2}\right)$-approximation to $E_{k, 0}$ reads

$$
E_{k, 0}=\frac{1}{2} \rho \int_{-\infty}^{\infty} \int_{-\infty}^{\infty}(\Phi)_{z=0} C d x d y+O\left(\mu^{2}\right)
$$

It is advantageous to write $C$ as a Laplacian, say $C=-\Delta B$. Substituting this expression in (29) we find that

$$
\left(1-\frac{1}{2} \mu h^{2} \Delta\right) \nabla B=h\left(1-\frac{1}{6} \mu h^{2} \Delta\right) \nabla \Phi(x, y, 0)
$$

Since $\Phi \rightarrow 0$ as $x^{2}+y^{2} \rightarrow \infty$, it follows that

$$
\begin{aligned}
E_{k, 0} & =-\frac{1}{2} \rho \int_{-\infty}^{\infty} \int_{-\infty}^{\infty} \Phi(x, y, 0) \Delta B \mathrm{~d} x \mathrm{~d} y+O\left(\mu^{2}\right) \\
& =\frac{1}{2} \rho \int_{-\infty}^{\infty} \int_{-\infty}^{\infty} \nabla \Phi(x, y, 0) \nabla B \mathrm{~d} x \mathrm{~d} y+O\left(\mu^{2}\right)
\end{aligned}
$$

which is upon substitution of (30) equal to Assertion 2. 
3. Employing a Taylor series expansion we find

$$
\nabla \Phi(x, y, 0) \approx \nabla \Phi(x, y, \varepsilon \mu \zeta)-\varepsilon \mu \zeta \nabla \Phi_{z}(x, y, \varepsilon \mu \zeta)
$$

Because $R$ is self-adjoint the difference in the proposition can be written as

$$
\frac{1}{2} \int_{-\infty}^{\infty} \int_{-\infty}^{\infty} \nabla\left(\Phi_{z=0}+\phi\right) R_{0} \nabla\left(\Phi_{z=0}-\phi\right) \mathrm{d} x \mathrm{~d} y
$$

Using (25) and (31) this equals

$$
-\frac{1}{2} \varepsilon \mu \int_{-\infty}^{\infty} \int_{-\infty}^{\infty} \nabla\left(\Phi_{z=0}+\phi\right) R_{0}\left(\nabla \zeta \Phi_{z}+\zeta \nabla \Phi_{z}\right)_{z=\varepsilon \mu \zeta} \mathrm{d} x \mathrm{~d} y
$$

which yields Assertion 3 .

During our research, we first derived $\mathcal{H}_{a p p}$ with $\alpha=0$ which is more or less a straight-forward approximation of $\mathcal{H}_{0}$. The precise steps in this approximation are described in the proof of the following theorem. The Hamiltonian is, as far as we can see, only positive in this case. At the end of the section we will comment further on this subject.

THEOREM 1. For $\alpha=0, \mathcal{H}_{\text {app }}-\mathcal{H}=O\left(\varepsilon \mu, \mu^{2}\right)$ and $\mathcal{H}_{\text {app }}$ is positive.

\section{Proof:}

From (23), it follows that $E_{k}$ can be approximated by

$$
E_{k} \approx \frac{1}{2} \rho \int_{-\infty}^{\infty} \int_{-\infty}^{\infty} \nabla \phi\left[R_{0}+\varepsilon \zeta\right] \nabla \phi \mathrm{d} x \mathrm{~d} y
$$

It is not clear whether this approximation of the kinetic energy is positive, but we can deduce an approximation from it which is. First we use that

$$
\begin{aligned}
R_{0} & =\left[\left(1-\frac{1}{2} \mu h^{2} \Delta\right)^{-1} h\left(1-\frac{1}{6} \mu h^{2} \Delta\right)\right] \\
& =\left[\left(1-\frac{1}{2} \mu h^{2} \Delta\right)^{-1} h\left(1+\frac{1}{6} \mu h^{2} \Delta\right)^{-1}\right]+O\left(\mu^{2}\right)
\end{aligned}
$$

The first term in the right-hand side is equal to

$$
\left[\left(1+\frac{1}{6} \mu h^{2} \Delta\right) h^{-1}\left(1-\frac{1}{2} \mu h^{2} \Delta\right)\right]^{-1}
$$

By neglecting another $O\left(\mu^{2}\right)$ term and after carrying out the differentiations involving $h$ the last expression becomes

$$
\left[h^{-1}-\frac{1}{6} \mu(h \Delta+\Delta(h \cdot))+\frac{1}{3} \mu h^{-1}|\nabla h|^{2}\right]^{-1}=\left[h^{-1}+\mu A\right]^{-1}
$$

where $A$ is the self-adjoint positive operator given by

$$
A=-\frac{1}{6}(h \Delta+\Delta(h \cdot))+\frac{1}{3} h^{-1}|\nabla h|^{2}
$$

Furthermore, we use the approximation

$$
\varepsilon \zeta+\left[h^{-1}+\mu A\right]^{-1}=\left[(h+\varepsilon \zeta)^{-1}+\mu A\right]^{-1}+O\left(\varepsilon \mu, \mu^{2}\right)
$$


Hence,

$$
R_{0}+\varepsilon \zeta=\left[(h+\varepsilon \zeta)^{-1}+\mu A\right]^{-1}+O\left(\varepsilon \mu, \mu^{2}\right)
$$

which proves the theorem.

In order to find a positive-definite approximation we introduce an extra degree of freedom.

THEOREM 2. For $\alpha>0, \mathcal{H}_{\text {app }}-\mathcal{H}=O\left(\varepsilon \mu, \mu^{2}\right)$ and $\mathcal{H}_{\text {app }}$ is positive definite.

Proof:

In this proof we will use $\breve{h}$ to denote $h+\varepsilon \zeta$. Now we write

$$
\left[\left(\check{h}^{-1}+\mu A\right]^{-1}=\sqrt{\check{h}}[I+\mu \sqrt{\check{h}} A \sqrt{\check{h}}]^{-1} \sqrt{\check{h}}\right.
$$

where the inverted operator in the right-hand side is also symmetric. We approximate the right-hand side by

$$
R=\sqrt{\breve{h}}\left[(I+\alpha \mu B)^{-1}(I+\beta \mu B)\right]^{-1} \sqrt{\check{h}}
$$

with $\beta=\alpha+1$ and $B=\sqrt{\check{h}} A \sqrt{\check{h}}$. This approximation satisfies

$$
R-\left[\breve{h}^{-1}+\mu A\right]^{-1}=O\left(\mu^{2}\right)
$$

Alternatively $R$ may be written as

$$
\begin{aligned}
R & =\sqrt{\breve{h}}(I+\beta \mu B)^{-1}(I+\alpha \mu B) \sqrt{\check{h}} \\
& =\sqrt{\check{h}}(I+\beta \mu B)^{-1}\left(\frac{1}{\beta} I+\frac{\alpha}{\beta}(I+\beta \mu B) \sqrt{\check{h}}\right. \\
& =\frac{1}{\beta} \sqrt{\check{h}}(I+\beta \mu B)^{-1} \sqrt{\check{h}}+\frac{\alpha}{\beta} \check{h} \\
& =\frac{1}{\beta}\left(\check{h}^{-1}+\beta \mu A\right)^{-1}+\frac{\alpha}{\beta} \check{h}
\end{aligned}
$$

which equals (20). It is easy to see that $R$ is a self-adjoint and positive-definite operator for $\alpha>0$.

The extra degree of freedom is used to approximate a next term of the linear dispersion relation for a horizontal bottom (cf. [6]). The linear dispersion relation (17) should be compared to the linear dispersion relation of our model at dimensionless depth $h=1$

$$
\tilde{c}^{2}=\hat{R}
$$

where the Fourier symbol of $R$ is given by

$$
\hat{R}=\frac{1}{\beta\left(1+\frac{1}{3} \beta \mu k^{2}\right)}+\frac{\alpha}{\beta}=\frac{1+\frac{1}{3} \alpha \mu k^{2}}{1+\frac{1}{3} \beta \mu k^{2}}
$$

In both cases we observe that for small $\sqrt{\mu} k$ the phase velocity of the shallow water equations is obtained, $c=1$ in dimensionless coordinates, which is independent of $k$. Due to the fact that a first-order approximation is derived, also the second term in the Taylor expansions of 
the respective phase velocities are equal for any $\alpha$ (recall that $\beta=1+\alpha$ ). The remaining freedom is used to match a next term of the expansions. Hence, we require that

$$
c^{2}-\tilde{c}^{2}=O\left((\sqrt{\mu} k)^{6}\right)
$$

which follows after an elementary calculation for $\alpha=1 / 5$.

Now we will address the stability question. Consider the metric

$$
\tilde{\mathrm{d}}(\nabla \phi, \zeta)=(\nabla \phi,(h+\varepsilon \zeta) \nabla \phi)+\|\zeta\|^{2}
$$

where $(\cdot, \cdot)$ and $\|\cdot\|$ denote the usual $L_{2}$ innerproduct and norm as in Equation (11), respectively. A straightforward calculation shows that (cf. Equation (8))

$$
\frac{\alpha}{\beta} \tilde{\mathrm{d}}(\nabla \phi, \zeta)<\mathcal{H}_{a p p}(\nabla \phi, \zeta) \leq \tilde{\mathrm{d}}(\nabla \phi, \zeta)
$$

Hence, reasoning similarly as in Section 2.2 , the zero state $\nabla \phi \equiv \zeta \equiv 0$ is stable for all $\alpha>0$. It may still be the case that by a sophisticated reasoning also $\alpha=0$ leads to a stable model. The above considerations yield only a sufficient condition for stability. On the other hand, also the exact Hamiltonian is only positive, which can be seen from the exact linear dispersion relation which yields a zero phase velocity for a wave number tending to infinity.

\section{The canonical equations corresponding to $\mathcal{H}_{a p p}$}

Having made an approximation, we can return to the original dimensions and derive the canonical equations. There the Hamiltonian $\mathcal{H}_{a p p}$ is given by

$$
\mathcal{H}_{a p p}=\frac{1}{2} \rho \int_{-\infty}^{\infty} \int_{-\infty}^{\infty} \nabla \phi\left[\frac{1}{\beta}\left((h+\zeta)^{-1}+\beta A\right)^{-1}+\frac{\alpha}{\beta}(h+\zeta)\right] \nabla \phi+g \zeta^{2} \mathrm{~d} x \mathrm{~d} y
$$

with $\beta=\alpha+1$. According to Section 2.3, we must calculate $\left(H_{a p p}\right)_{\zeta}$ and $\left(H_{a p p}\right)_{\phi}$. The first is implicitly given by

$$
\begin{aligned}
\left(\left(H_{a p p}\right)_{\zeta}, v\right)= & \lim _{\epsilon \rightarrow 0} \frac{\mathcal{H}_{a p p}(\phi, \zeta+\epsilon v)-\mathcal{H}_{a p p}(\phi, \zeta)}{\epsilon} \\
= & \frac{1}{2} \rho \int_{-\infty}^{\infty} \int_{-\infty}^{\infty} \nabla \phi \frac{1}{\beta} \lim _{\epsilon \rightarrow 0} \frac{1}{\epsilon}\left(\left[(\check{h}+\epsilon v)^{-1}+\beta A\right]^{-1}-\left[\check{h}^{-1}+\beta A\right]^{-1}\right) \nabla \phi \\
& +v \frac{\alpha}{\beta}|\nabla \phi|^{2}+g \zeta v \mathrm{~d} x \mathrm{~d} y
\end{aligned}
$$

where we have used $\breve{h}$ to denote $h+\zeta$. Now, we deduce that

$$
\begin{aligned}
& {\left[(\breve{h}+\epsilon v)^{-1}+\beta A\right]^{-1}-\left[\check{h}^{-1}+\beta A\right]^{-1}} \\
& \quad=\left[\check{h}^{-1}+\beta A\right]^{-1}\left(\check{h}^{-1}-(\check{h}+\epsilon v)^{-1}\right)\left[(\check{h}+\epsilon v)^{-1}+\beta A\right]^{-1} \\
& \quad=\epsilon\left[\check{h}^{-1}+\beta A\right]^{-1} \frac{v}{\check{h}(\check{h}+\epsilon v)}\left[(\check{h}+\epsilon v)^{-1}+\beta A\right]^{-1} \\
& \quad=\epsilon R_{\beta}\left[\check{h}^{-2} v R_{\beta}\right]+O\left(\epsilon^{2}\right)
\end{aligned}
$$

with $R_{\beta}$ given by

$$
R_{\beta}=\left[(h+\zeta)^{-1}+\beta A\right]^{-1}
$$


Using this result we see that

$$
\left(\left(H_{\text {app }}\right)_{\zeta}, v\right)=\frac{1}{2} \rho \int_{-\infty}^{\infty} \int_{-\infty}^{\infty}\left[\frac{1}{\beta}\left|(h+\zeta)^{-1} R_{\beta} \nabla \phi\right|^{2}+\frac{\alpha}{\beta}|\nabla \phi|^{2}+2 g \zeta\right] v \mathrm{~d} x \mathrm{~d} y
$$

where the symmetry of $R_{\beta}$ is used and hence $\left(H_{a p p}\right)_{\zeta}$ is given by

$$
\left(H_{a p p}\right)_{\zeta}=\frac{1}{2} \rho\left[\frac{1}{\beta}\left|(h+\zeta)^{-1} R_{\beta} \nabla \phi\right|^{2}+\frac{\alpha}{\beta}|\nabla \phi|^{2}+2 g \zeta\right]
$$

The derivation of $\left(H_{a p p}\right)_{\phi}$ proceeds as follows:

$$
\begin{aligned}
\left(\left(H_{a p p}\right)_{\phi}, v\right) & =\lim _{\epsilon \rightarrow 0} \frac{\mathcal{H}_{a p p}(\phi+\epsilon v, \zeta)-\mathcal{H}_{a p p}(\phi, \zeta)}{\epsilon} \\
& =\rho \int_{-\infty}^{\infty} \int_{-\infty}^{\infty} \nabla v R \nabla \phi \mathrm{d} x \mathrm{~d} y
\end{aligned}
$$

where

$$
R=\frac{1}{\beta}\left(R_{\beta}+\alpha(h+\zeta)\right)
$$

Applying Green's theorem we obtain

$$
\left(\left(H_{a p p}\right)_{\phi}, v\right)=-\rho \int_{-\infty}^{\infty} \int_{-\infty}^{\infty} v \operatorname{div}(R \nabla \phi) \mathrm{d} x \mathrm{~d} y
$$

Hence, the canonical equations are according to (9) and (10) given by

$$
\begin{aligned}
\phi_{t} & =-\frac{1}{2 \beta}\left|(h+\zeta)^{-1} R_{\beta} \nabla \phi\right|^{2}-\frac{\alpha}{2 \beta}|\nabla \phi|^{2}-g \zeta \\
\zeta_{t} & =-\operatorname{div}(R \nabla \phi)
\end{aligned}
$$

These are the desired equations and they are used as a starting-point for a numerical model. Note that for the evaluation of the right-hand side, we need to compute $R \nabla \phi$ and $R_{\beta} \nabla \phi$. From (34) it follows that $R \nabla \phi$ is composed of $R_{\beta} \nabla \phi / \beta$ plus a simple explicit operation. The computation of $f=R_{\beta} \nabla \phi$ is in fact the solution of the problem $\left.(h+\zeta)^{-1}+\beta A\right) f=\nabla \phi$, which is of Helmholtz type. Hence, only one Helmholz-type equation needs to be solved for the right-hand side evaluation.

At this place, it is in order to compare our approximate Hamiltonian to the one proposed by Mooiman based on the work of Broer. That Hamiltonian reads

$$
\mathcal{H}_{a p p}=\frac{1}{2} \rho \int_{-\infty}^{\infty} \int_{-\infty}^{\infty}(h+\zeta)(G \nabla \phi)^{2}+g \zeta^{2} \mathrm{~d} x \mathrm{~d} y
$$

where $G$ is a rational expression like $R$ but now independent of $\zeta$. Of course, $G^{*}(h+\zeta) G=$ $R+O\left(\varepsilon \mu, \mu^{2}\right)$, where $G^{*}$ is the adjoint of $G$. The derivation of the equations is as described in this section. However, instead of $R$ we will find $G^{*}(h+\zeta) G$ in the continuity equation. The application of this operator requires twice the solution of a Helmholz-type equation. This factor two is actually found by comparing the run time of the two models on a vector computer (NEC SX-3) using comparable numerical techniques. Furthermore, some experiments carried out by experts in the field indicate that our model is at least as accurate as that of Mooiman [5]. 


\section{Concluding remarks}

The propagation of nonlinear dispersive gravity waves is described by a Hamiltonian system in which the elevation and the velocity potential at the free surface are the canonical variables. However, the corresponding canonical equations contain a boundary integral operator, which makes the numerical solution process expensive. For fairly low and fairly long waves a class of of first-order approximations to this Hamiltonian can be found, for which the canonical equations contain a numerically attractive approximation to this boundary integral.

In order to find a stable Boussinesq model, it is sufficient that the approximate Hamiltonian is positive-definite. The difficult part is the kinetic energy. This energy is split in a part above and a part below the still-water level. The part above the still-water level is easily approximated. By Greens' formula one can obtain a boundary integral of the part below the still-water level, containing the product of the velocity potential and its normal derivative. Due to the impermeability of the bottom there is only a contribution at the still-water level. The normal derivative is expressed in terms of a simple integral operator acting only in tangential directions using Taylor series and the impermeability condition at the bottom. Next the two parts of the kinetic energy are recombined. The part above the still-water level can be included by modifying the integral operator slightly. Moreover, we have altered it such that it becomes self-adjoint. The method is optimal in the sense that only once per right-hand side evaluation a Helmholtz-type equation needs to be solved, which is a reduction of a factor two compared to the method proposed by Mooiman based on the work of Broer.

Further we were able, like in Mooiman's method, to choose our approximation such that the first three terms in the Taylor series expansions of our dispersion relation for even bottoms and that of the exact dispersion relation are equal, which appeared to be advantageous in computations.

In this paper, we gave no numerical results of our model. An implementation has been made already in $(u, v, \zeta)$ variables, as in Mooiman's model. The method is stable and the reduction of computation time with respect to Mooiman's model is more than a factor two, as expected. Currently, tests are performed in order to validate the quality of the model. First results indicate that the accuracy of our model is at least as good as that of Mooiman's model.

\section{Appendix}

\section{A. Expressing $E_{k, 0}$ as function of $\nabla \Phi(x, y, 0)$}

Here, we present an exact expression for $E_{k, 0}$ as a function of $\nabla \Phi(x, y, 0)$ which may be of interest if higher-order approximations have to be derived. In view of (26) we must express $\Phi_{z}(x, y, 0)$ as a function of $\nabla \Phi(x, y, 0)$. First we consider the Taylor series expansion of $\Phi(x, y, z)$

$$
\begin{aligned}
\Phi(x, y, z)= & \sum_{k=0}^{\infty} \frac{1}{k !} \frac{\partial^{k}}{\partial z^{k}} \Phi(x, y, 0) z^{k} \\
= & \sum_{k=0}^{\infty} \frac{1}{(2 k) !}\left(-\frac{1}{\mu}\right)^{k} \Delta^{k} \Phi(x, y, 0) z^{2 k} \\
& +\sum_{k=0}^{\infty} \frac{1}{(2 k+1) !}\left(-\frac{1}{\mu}\right)^{k} \Delta^{k}\left(\Phi_{z}(x, y, 0)\right) z^{2 k+1}
\end{aligned}
$$


For convenience we introduce the functions

$$
\varphi(x, y)=\Phi(x, y, 0), \quad \varphi_{z}(x, y)=\Phi_{z}(x, y, 0)
$$

and the linear operators

$$
F(z) u=\sum_{k=0}^{\infty} \frac{1}{(2 k) !}\left(-\frac{1}{\mu}\right)^{k} z^{2 k} \Delta^{k} u
$$

and

$$
G(z) u=\sum_{k=0}^{\infty} \frac{1}{(2 k+1) !}\left(-\frac{1}{\mu}\right)^{k} z^{2 k+1} \Delta^{k} u
$$

Then the Taylor Series expansion can be written as

$$
\Phi(x, y, z)=F(z) \varphi(x, y)+G(z) \varphi_{z}(x, y)
$$

The operators $F$ and $G$ have the following properties:

$$
\begin{aligned}
F(-z) & =F(z) \\
G(-z) & =-G(z) \\
\frac{\partial G}{\partial z} & =F \\
\frac{\partial F}{\partial z} & =-\frac{1}{\mu} G \Delta
\end{aligned}
$$

Substituting (35) in the impermeability condition at the bottom

$$
\nabla \Phi \cdot \nabla h+\Phi_{z}=0, \quad z=-\mu h(x, y)
$$

gives

$$
\left[F(\mu h) \nabla \varphi-G(\mu h) \nabla \varphi_{z}\right] \nabla h-\frac{\partial F}{\partial z}(\mu h) \varphi+\frac{\partial G}{\partial z}(\mu h) \varphi_{z}=0
$$

To simplify this equation, we first note that

$$
\begin{aligned}
-\frac{\partial F}{\partial z}(\mu h) \varphi+(F(\mu h) \nabla \varphi) \cdot \nabla h & =\frac{1}{\mu} G(\mu h) \Delta \varphi+\nabla h \cdot\left(\frac{\partial G}{\partial z}(\mu h) \nabla \varphi\right) \\
& =\frac{1}{\mu} \operatorname{div}(G(\mu h) \nabla \varphi)
\end{aligned}
$$

and

$$
\begin{aligned}
\frac{\partial G}{\partial z}(\mu h) \varphi_{z} & -\left(G(\mu h) \nabla \varphi_{z}\right) \cdot \nabla h \\
& =F(\mu h) \Delta^{-1} \Delta \varphi_{z}-G(\mu h) \nabla \varphi_{z} \cdot \nabla h \\
& =(F(\mu h)-1) \Delta^{-1} \Delta \varphi_{z}+\varphi_{z}+\mu \nabla h \cdot \frac{\partial F}{\partial z}(\mu h) \Delta^{-1} \nabla \varphi_{z} \\
& =\left(1+\operatorname{div}(F(-\mu h)-1) \Delta^{-1} \nabla\right) \varphi_{z}
\end{aligned}
$$


Using these identities in (36), we obtain

$$
\left(1+\operatorname{div}(F(\mu h)-1) \Delta^{-1} \nabla\right) \varphi_{z}=-\frac{1}{\mu} \operatorname{div}(G(\mu h) \nabla \varphi)
$$

Under mild conditions, there is a $B$ such that $\varphi_{z}=-\Delta B$. Herewith, we readily obtain the following equation for $B$ (cf. (30))

$$
F(\mu h) \nabla B=\frac{1}{\mu} G(\mu h) \nabla \varphi
$$

With this result the kinetic energy can be written as

$$
\begin{aligned}
E_{k, 0} & =\int_{-\infty}^{\infty} \int_{-\infty}^{\infty} \varphi \varphi_{z} \mathrm{~d} x \mathrm{~d} y \\
& =\int_{-\infty}^{\infty} \int_{-\infty}^{\infty} \nabla B \nabla \varphi \mathrm{d} x \mathrm{~d} y \\
& =\int_{-\infty}^{\infty} \int_{-\infty}^{\infty} \nabla \varphi(F(\mu h))^{-1}\left(\frac{1}{\mu} G(\mu h) \nabla \varphi\right) \mathrm{d} x \mathrm{~d} y
\end{aligned}
$$

Neglecting $O\left(\mu^{2}\right)$ terms yields Assertion 2 of Lemma 1 as expected.

\section{References}

1. R. Abraham and J. E. Marsden. Foundations of Mechanics : A Mathematical Exposition of Classical Mechanics with an Introduction to the Qualitative Theory of Dynamical Systems and Applications to the Three-Body Problem. Benjamin/Cummings Publishing Co, (1978).

2. T.B. Benjamin. Impulse, flow, force and variational principles. IMA Journal of applied Mathematics 32 (1984) 3-68.

3. T.B. Benjamin and P.J. Olver. Hamiltonian structure, symmetries and conservation laws for waterwaves. $J$. Fluid Mech. 125 (1982) 137-185.

4. L.J.F. Broer. On the hamiltonian theory of surface waves. Appl. Sci. Res. 30 (1974) $430-446$.

5. M.W. Dingemans. Comparison of computations with Boussinesq-like models and laboratory measurements. MAST-G8M note, in preparation.

6. E.W.C. van Groesen L.J.F. Broer and J.M.W. Timmers. Stable model equations for long water waves. Appl. Sci. Res., 32, (1976) 619-636.

7. J. Mooiman. Boussinesq equations based on a positive definite Hamiltonian. Technical report, Delft Hydraulics, (February 1991). Report Z294.

8. J. Mooiman and G.K. Verboom. A new Boussinesq model based on a positive definite Hamiltonian. (1992). Proceedings of the "IX International Conference on Computational Methods in Water Resources", Denver.

9. A. van der Ploeg and F.W. Wubs. Vectorizable preconditioning techniques for solving the Boussinesq equations. In Ch. Hirsch, J. Periaux, and W. Kordulla, editors, Computational Fluid Dynamics '92, Elsevier, (1992) $481-488$.

10. G.B. Witham. Linear and Nonlinear Waves. Wiley-Interscience, New York, (1974). 